\title{
Analysis of phase-resolved partial discharge patterns of voids based on a stochastic process approach
}

\author{
R Altenburger ${ }^{1}$, C Heitz ${ }^{2}$ and J Timmer ${ }^{1}$ \\ ${ }^{1}$ Freiburg Center of Data Analysis and Modeling, Germany \\ ${ }^{2}$ Institute of Data Analysis and Process Design, Zurich University of Applied Sciences \\ Winterthur, Switzerland
}

Received 28 November 2001, in final form 3 April 2002

Published 21 May 2002

Online at stacks.iop.org/JPhysD/35/1149

\begin{abstract}
A method is presented for the determination of physical discharge parameters for partial discharges (PDs) of voids in solid insulation. Based on a recently developed stochastic theory of PD processes, a statistical analysis of a measured phase-resolved partial discharge (PRPD) pattern allows the determination of the relevant physical parameters like first electron availability or decay time constants for deployed charge carriers. These parameters can be estimated directly from the measured patterns without the need of performing simulations. Furthermore, error bounds for the parameter values can be given.

The parameter estimation algorithm is based on the analysis of a contiguous region of the PRPD pattern where this region can be chosen nearly arbitrarily. Thus, even patterns with several active PD defects or patterns which are corrupted by noise can be analysed.

The method is applied to a sequence of patterns of a void in epoxy resin. The change in first electron availability in the course of a day can be determined quantitatively from the data while the other physical parameters remain constant.
\end{abstract}

\section{Introduction}

Much work has been established in the last two decades in order to understand and describe the nature of partial discharge (PD) processes [1-9]. For some discharge phenomena physical models have been developed (e.g. [2,5,22,27]). These models have substantially increased the knowledge of the physical processes during PD processes. For the analysis and interpretation of measured PD data, this knowledge is fundamental. It allows to extract information about the physical situation at the PD defect.

On the other hand, different PD measuring systems have been developed which allow the detection and recording of PDs. These systems are now widely used for monitoring and failure detection in high-voltage equipment. Especially phaseresolved partial discharge (PRPD) measurement systems have become very popular.

However, the analysis and interpretation of measured PD data is still a difficult task. Many publications treat this problem (e.g. [10-29]). Two different kinds of analysis methods are used: the first method is based on a description of the PD data by means of a set of parameters which, in most cases, are statistical features [13-21], e.g. the number of discharges per period, skewness and kurtosis of the pulse height or phase-distribution, and others. These parameters yield a characterization of the PD pattern and can thus be used for interpretation and classification tasks. The main disadvantage of such a purely descriptive characterization is the fact that the parameters are not physical parameters, making an interpretation difficult. For example, an increase of the applied voltage may lead to other values of the statistical parameters, even if the physics of the discharge does not change significantly.

In contrast, the second analysis method is based on a physical model which is used to perform simulations of the PD process. Comparison of the simulated data with the measured ones allows for the determination of the physical parameters. Examples of this approach are [22-29]. This approach renders a characterization of the pattern by physical parameters 
which, in most cases, is more useful than a purely descriptive characterization. However, the simulation-comparison method is difficult to use since there is no systematic way to find the correct parameter settings. Most often, the parameters are found by trial and error. This prevents the physical method to be used in automatic pattern analysis algorithms.

In this paper, a new method is presented which allows the extraction of physical discharge parameters from measured PD data in a systematic and automatic way, based on the analysis of PRPD patterns. It is based on a newly developed description of the PD process [8] which is consequently formulated in a stochastic framework. The discharge process is assumed to be governed by few pysical parameters the most important ones are the first electron availability and the charge removal after a discharge.

By statistical data analysis of the measured pattern, the values of the physical parameters can be extracted directly from a measured PRPD pattern. Thus, a characterization by physical parameters can be obtained without the need of simulation. This overcomes one of the major restrictions of the above-mentioned physically based analysis methods. It thus combines the advantage of the purely descriptive method (possibility of automated parameter extraction) with the advantage of the physical model-based approach (characterization by physically meaningful parameters).

The method is explicitely worked out for the case of void discharges with constant first electron supply rate. However, as will be shown in section 3.6, it can be extended to nonconstant hazard rate. Furthermore, the idea of the parameter estimation method could be used for other kinds of discharges like surface discharges or corona discharges.

The new method of data analysis has the advantageous property that it can be applied even to small parts of the pattern. Thus, it is possible to analyse separately different PD sources which are separated in the PRPD pattern. Furthermore, it is possible to deal with disturbances which, in practice, cannot be avoided. Therefore, the method is not restricted to the laboratory but can be used in the daily engineering work.

The paper is structured as follows: in section 2 the description of the PD process as a stochastic process will be reviewed briefly. In sections 3 and 4 the new method of data analysis is described. In section 5 the method is applied to simulated data, while in section 6 the application to measured PD patterns is shown.

\section{PD process as a stochastic process}

The presented pattern analysis method is based on a recently developed description of the PD process as a stochastic process which will be reviewed briefly in the following. For details, the reader is referred to the original paper [8]. Within this paper, the discussion is restricted to the case of void discharges. However, the original model is more general and can be applied to other kinds of discharges as well.

The PD process consists of the sequence of electrical discharges under an externally applied electrical field $E_{0}(t)$. The discharges (PD events) lead to a charge deployment in the vicinity of the PD defect which give rise to a so-called internal field $E_{\mathrm{i}}(t)$. Each PD event changes the internal field suddenly. Between the successive PD events, the internal field changes due to charge dissipation mechanisms like surface conduction in voids, or charge carrier drift in gas discharges. The PD process itself can thus be described by the evolution of the internal field $E_{\mathrm{i}}(t)$. The dynamics of this process is described briefly in the following.

During a discharge at time $t$ the total electric field $E_{\mathrm{tot}}(t)$,

$$
E_{\mathrm{tot}}(t)=E_{0}(t)+E_{\mathrm{i}}(t),
$$

drops to a residual field $\mathrm{E}_{\text {res }}$ :

$$
E_{\mathrm{tot}} \mapsto E_{\mathrm{tot}}^{\prime}= \pm E_{\mathrm{res}} .
$$

The positive sign is chosen if $E_{\mathrm{tot}}(t)>0$ and vice versa. The value $E_{\text {res }}$ of the residual field is defect specific. It is assumed that the residual field has a constant value for each discharge.

With equation (2) a discharge leads to a sudden jump in $E_{\mathrm{i}}$ :

$$
E_{\mathrm{i}}(t) \mapsto E_{\mathrm{i}}^{\prime}(t)= \pm E_{\mathrm{res}}-E_{0}(t) .
$$

The bipolar charge distribution deployed in the vicinity of the PD defect tends to vanish by drift and recombination processes. In general, it can be described by a differential equation:

$$
\dot{E}_{\mathrm{i}}(t)=f\left(E_{\mathrm{i}}(t), E_{0}(t)\right) .
$$

In contrast to the jump process during a PD event, this field change is continuous and deterministic until the next PD event takes place. A simplification consists in setting

$$
f=-E_{\mathrm{i}} / \tau
$$

leading to an exponential decay with a single time constant $\tau$ which does not depend on $E_{\mathrm{i}}$ and $E_{0}$ :

$$
E_{\mathrm{i}}(t)=E_{\mathrm{i}}\left(t_{0}\right) \mathrm{e}^{-t / \tau} .
$$

In this simplification, the drift and recombination process is subsumed by a single parameter $\tau$ which is a time constant for the charge decay process. Note that this only can be a very rough approximation to the real physical drift and recombination processes which cannot take into acount the detailed physical processes. Examples are field-dependent surface conduction, or shielding effects due to conductivity of cavity walls. On the other hand, the parameter $\tau$ has a direct physical meaning. For different kinds of discharges, the time constant for the decay of internal charge can be very different. For example, for a void discharge, where the internal charge may decay by surface conduction, the time constant is of the order of magnitude of minutes or hours.

Both processes, discharge (jump of $E_{\mathrm{i}}$ ) and drift/ recombination (continuous change of $E_{\mathrm{i}}$ ), interact in a real PD process. They are coupled by the discharge probability in the following way: let $c \mathrm{~d} t$ be the probability that a fast discharge occurs in the time interval $[t, t+\mathrm{d} t]$. This probability may depend on the internal field $E_{\mathrm{i}}(t)$ as well as on the external field $E_{0}(t)$ and depends on the mechanism of first electron supply [8]. For a void where starting electrons are supplied mainly by external radiation, we have $c=0$ for $E_{\text {tot }}<E_{\text {inc }}$ with a typical inception field $E_{\text {inc }}$, and $c=$ const for $E_{\text {tot }}>E_{\text {inc }}$. The value of $c$ depends on the void volume and the electron production rate of the externally imposed radiation [5]. 
For a small time step $\mathrm{d} t$ the time evolution of $E_{\mathrm{i}}(t)$ is shown schematically in figure 1 . Two different paths are possible: a fast discharge, or a slow discharge due to drift and recombination. The probability for each path is $c \mathrm{~d} t$ and $1-c \mathrm{~d} t$, respectively. In figure 2 typical (random) trajectories of $E_{\mathrm{tot}}(t)$ and $E_{\mathrm{i}}(t)$ for the case of AC voltage are shown. Note that the same process realization is shown in figures $2(a)$ and $(b)$.

The dynamics of the internal field forms a piecewise deterministic Markov process [30]. Such processes are generally described by a dynamical equation for the timedependent probability density $p\left(E_{\mathrm{i}} ; t\right)$. From the short-time dynamics of the stochastic process as sketched in figure 1 , a differential equation for $p\left(E_{\mathrm{i}} ; t\right)$ can be constructed, the so-called master equation [31]. For the considered PD system one gets (see [8] for details)

$$
\begin{aligned}
\frac{\partial}{\partial t} p & \left(E_{\mathrm{i}} ; t\right)=-c\left(E_{\mathrm{i}}, E_{0}(t)\right) \cdot p\left(E_{\mathrm{i}} ; t\right) \\
& -\frac{\partial}{\partial E_{\mathrm{i}}}\left(f\left(E_{\mathrm{i}}, E_{0}(t)\right) \cdot p\left(E_{\mathrm{i}} ; t\right)\right) \\
& +\left(\int_{E_{\mathrm{i}}^{\prime}+E_{0}(t)>0} c\left(E_{\mathrm{i}}^{\prime}, E_{0}(t)\right) \cdot p\left(E_{\mathrm{i}}^{\prime} ; t\right) \mathrm{d} E_{\mathrm{i}}^{\prime}\right) \\
& \cdot \delta\left(E_{\mathrm{i}}-\left(E_{\mathrm{res}}-E_{0}(t)\right)\right) \\
& +\left(\int_{E_{\mathrm{i}}^{\prime}+E_{0}(t)<0} c\left(E_{\mathrm{i}}^{\prime}, E_{0}(t)\right) \cdot p\left(E_{\mathrm{i}}^{\prime} ; t\right) \mathrm{d} E_{\mathrm{i}}^{\prime}\right) \\
& \cdot \delta\left(E_{\mathrm{i}}-\left(-E_{\mathrm{res}}-E_{0}(t)\right)\right),
\end{aligned}
$$

where $\delta(\cdot)$ is the Kronecker delta function.

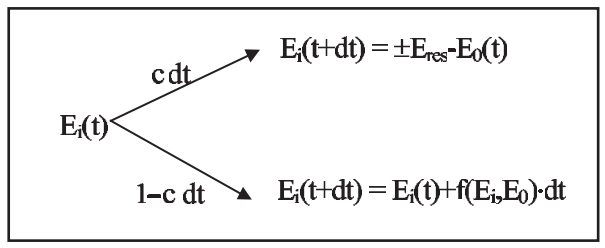

Figure 1. Time evolution of $E_{\mathrm{i}}(t)$ to $E_{\mathrm{i}}(t+\mathrm{d} t)$ for small $\mathrm{d} t$. The upper path with probability $c \mathrm{~d} t$ represents the change of $E_{\mathrm{i}}(t)$ due to a discharge, the lower path with probability $1-c \mathrm{~d} t$ is the time development for the case of no discharge.

(a)

(b)

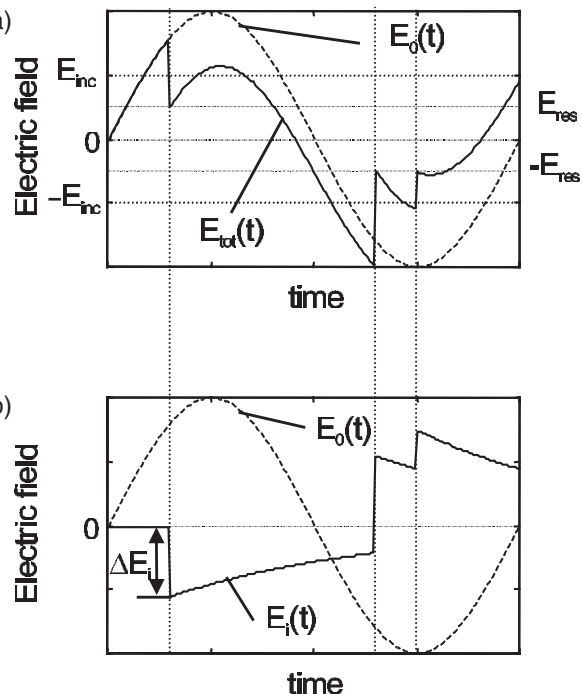

Figure 2. Typical trajectories under $\mathrm{AC}$ for $(a) E_{\mathrm{tot}}(t)=$ $E_{0}(t)+E_{\mathrm{i}}(t)$ and $(b) E_{\mathrm{i}}(t)$.
From $p\left(E_{\mathrm{i}} ; t\right)$ one can derive the probability density $p_{\mathrm{d}}\left(E_{\mathrm{i}}, t\right)$ of a PD event at time $t$ and internal field $E_{\mathrm{i}}$ (the subscript d stands for discharge)

$$
p_{\mathrm{d}}\left(E_{\mathrm{i}} ; t\right)=c\left(E_{\mathrm{i}}, E_{0}(t)\right) \cdot p\left(E_{\mathrm{i}} ; t\right) .
$$

Until now, the dynamics of the PD system has been formulated in terms of the internal field $E_{\mathrm{i}}(t)$. However, with the usual PD measuring technique $E_{\mathrm{i}}(t)$ cannot be measured, i.e. the PD process cannot be observed directly. Instead, the charge separation of the bipolar charge quantity $\pm q$ being deployed during the discharge is measured.

The jump $\Delta E$ of the internal field during a fast discharge according to equation (3) is given by

$$
\Delta E=E_{\mathrm{i}}^{\prime}-E_{\mathrm{i}}= \pm E_{\mathrm{res}}-\left(E_{\mathrm{i}}+E_{0}(t)\right) .
$$

This field change is accomplished by the deployment of a bipolar charge quantity which, for a void discharge, is proportional to the field change [8]. The measured charge $q$, in turn, is proportional to the deployed charge, leading to

$$
q=-\gamma \cdot \Delta E=\gamma\left(E_{\mathrm{i}}+E_{0}(t) \mu E_{\mathrm{res}}\right) .
$$

The proportionality factor $\gamma$ not only depends on the void geometry but also on the electromagnetic coupling of the void to the PD detector $[37,38]$. Given the field $E_{\mathrm{i}}$ just before the discharge and the external field $E_{0}$ at the discharge time, the charge $q$ can be calculated. The minus sign in the first line of equation (9) accounts for the usual convention that a charge is considered positive if the field change is negative, and vice versa.

The probability density $p_{\mathrm{d}}\left(E_{\mathrm{i}} ; t\right)$ can be transformed into the probability density $\tilde{p}_{\mathrm{d}}(q, t)$ of a discharge with specified charge $q$ at time $t$ :

$$
\tilde{p}_{\mathrm{d}}(q, t)=p_{\mathrm{d}}\left(E_{\mathrm{i}}(q), t\right) \frac{\mathrm{d} E_{\mathrm{i}}}{\mathrm{d} q} .
$$

For AC voltages, the so-called PRPD patterns correspond to $\tilde{p}_{\mathrm{d}}(q, t)$ for $t \in[0, T]$ or $\tilde{p}(q, \varphi)$ for $\varphi \in[0,2 \pi]$, respectively. When measuring a PRPD pattern, one plots each measured PD event $(t, q)$ in the $\varphi-q$ plane, where $\varphi$ is the phase angle of the discharge time $t$. Thus, the point density of a PRPD pattern directly corresponds to the probability density $\tilde{p}_{\mathrm{d}}(q, t)$ of the generating PD process. This yields the possibility of extracting the physical parameters of the model from measured PRPD data.

\section{Determination of parameters from measured PRPD data}

In this section the new method for extracting the physical parameters of the discharge directly from the measured PRPD pattern is presented. This method is described for the case of void discharges with a constant rate of initial electrons. It can be extended to the case of non-constant hazard rate as will be discussed in section 3.6

We consider the case of sinusoidal external voltage, leading to a field

$$
E_{0}(t)=\hat{E}_{0} \sin (\omega t)
$$


The considered model parameters which are estimated from measured PRPD data are,

- the time constant $\tau$ for the drift/recombination of deployed charge,

- the parameter $c$ which specifies the first electron availability,

- the maximum field $\hat{E}_{0}$ at the discharge site, and

- the residual field $E_{\text {res }}$

These parameters specify the dynamics of the stochastic discharge process. Three remarks are to be made:

1. The absolute values of the fields $\hat{E}_{0}, E_{\text {res }}$ and $E_{\text {inc }}$ cannot be derived from the PRPD measurements without a complete knowledge of the whole electric assembly (geometry, dielectric constants). However, for the dynamics of the process it suffices to know the fields $\hat{E}_{0}$ and $E_{\text {res }}$ in units $E_{\text {inc }}$. We therefore set $E_{\mathrm{inc}}=1$. The task is then to determine the value of $\hat{E}_{0}$ and $E_{\text {res }}$.

2. The absolute measured charge value $q$ for a PD event is proportional to the field change $\Delta E$ via a proportionality factor $\gamma$ which often is unknown. The analysis does not exploit the absolute value of the charge but only takes into account the internal structure of the PRPD pattern. Therefore it is not necessary to specify the proportionality factor $\gamma$ of equation (9).

3. For the following, we assume $c=$ constant for $E_{\text {tot }}$ above the inception field. Thus, we focus on the case of a constant rate of initial electrons. The case of initial electron supply by surface charge detrapping processes is not covered. In general, however, the electron supply may depend on the number of trapped charge carriers and the applied field. The model of [8] accounts for this case since $c$ may be a function of $E_{\mathrm{i}}$ and $E_{0}(t)$. It will be discussed in section 3.6 how the parameter extraction method can be extended to this more general case.

\subsection{General remarks on identifiability}

The parameters characterizing the physical situation of a void discharge are the charge decay time constant $\tau$ and the discharge probability density $c$, where $\tau$ is assumed to be constant, and $c$ is assumed to be constant for $E_{\text {tot }}>1$ (in units of $E_{\text {inc }}$ ), and zero otherwise.

These parameters have to be estimated from measured PRPD data. This is possible in principle because the discharge process itself depends on the parameter values. However, there are situations where a quantitative determination of the process parameters may not be possible. Two examples will illustrate this.

As a first example consider a discharge with $\tau$ being much larger than $1 / c$. Since $1 / c$ is a typical time interval between two successive discharges, this means that the internal field does not decay substantially between two successive discharges. This leads to the fact that the process itself is not dependent on the value of $\tau$, as long as $\tau$ is suffiently large. Consequently, the value of $\tau$ cannot be estimated quantitatively from the measured data. In other words, the data do not bear any information about $\tau$ except that $\tau$ is much larger than $1 / c$. In this case, a parameter estimation of $\tau$ can give only a lower bound for $\tau$. The exact value of $\tau$ cannot be determined.
As a second example, consider the case where $1 / c$ is much lower than the period time $T=2 \pi / \omega$ of the external field. This is the case if many first electrons are available. As soon as the electric field $E_{\text {tot }}$ at the defect site increases over the inception field $E_{\text {inc }}$, a discharge will take place. Typically this leads to PRPD patterns with horizontal lines; all discharges have the same field change $\Delta E$ and, consequently, the same charge. In this case no quantitative information about $c$ can be expected from the data except that $c$ is very large. Furthermore, if $\tau$ is larger than the period time of the driving external field, the value of $\tau$ cannot be determined reliably because the time interval between successive PD events does not exceed the period time $T$.

These examples show that under certain circumstances one or several of the process parameters cannot be estimated reliably from the measured data. Only a lower or an upper bound for the parameter can be given. Note that this is not a consequence of the used data analysis method. It is a consequence of the fact that the corresponding information is not present in the data. Therefore, a parameter extraction algorithm should not only give an estimated value of the physical parameter but also a confidence interval. Large confidence intervals indicate that there is a lack of information in the data.

\subsection{Estimation of the process parameters}

As shown in the last section, the PD process is governed by equation (6) which describes the temporal evolution of the density $p\left(E_{\mathrm{i}} ; t\right)$. Each $E_{\mathrm{i}}$ is linked to a charge $q$ by equation (9) which enables the transformation of the density $p\left(E_{\mathrm{i}} ; t\right)$ into a density $\tilde{p}_{\mathrm{d}}(q, t)$ describing the measured PD pattern of the process. The structure of $p_{\mathrm{d}}\left(E_{\mathrm{i}} ; t\right)$ or $\tilde{p}_{\mathrm{d}}(q, t)$, respectively, bears the information on the process parameters. However, the link between process parameters and the structure of the resulting density is rather complicated and cannot be given explicitely.

In order to simplify the equations, the data analysis is restricted to a contiguous region in the PRPD pattern where $c>0$ everywhere. Since each point $(q, t)$ is linked to a point $\left(E_{\mathrm{i}}, t\right)$ in the $E_{\mathrm{i}}-t$ space, this means that the analysis is restricted to a region in the $E_{\mathrm{i}}-t$ space, where

$$
\left|E_{\text {tot }}\right|>E_{\text {inc }} \text {. }
$$

This, in turn, has the consequence that the $\delta(\cdot)$ terms in equation (6) can be omitted (note that since $E_{\mathrm{res}}<E_{\mathrm{inc}}$, the argument of the $\delta$-terms cannot be zero for $\left.\left|E_{\text {tot }}\right|>E_{\text {inc }}\right)$. This leads to the following simplified equation for $p\left(E_{\mathrm{i}} ; t\right)$ :

$$
\frac{\mathrm{d}}{\mathrm{d} t} p=-c p-\frac{\partial}{\partial E_{\mathrm{i}}}(f p),
$$

where the arguments have been omitted for the ease of notation.

With $f=-E_{\mathrm{i}} / \tau$, one gets a simplified version of the master equation

$$
\frac{\mathrm{d}}{\mathrm{d} t} p=\left(\frac{1}{\tau}-c\right) p-\frac{E_{\mathrm{i}}}{\tau} \frac{\partial p}{\partial E_{\mathrm{i}}} .
$$

In a second step, instead of regarding the process in the $E_{\mathrm{i}}-t$ space, a transformation in an $x-t$ space is performed where the new variable $x$ is given by

$$
x=E_{\mathrm{i}} \mathrm{e}^{t / \tau} .
$$


Under this transformation, the dynamical equation (equation (13)) transforms to the simple form (see appendix A)

$$
\frac{\mathrm{d}}{\mathrm{d} t} \hat{p}(x ; t)=-c(x, t) \hat{p}(x ; t),
$$

where $\hat{p}(x ; t)$ is the time-dependent probability density function of the variable $x$. This equation can be solved analytically. For the case of void discharges where $c=$ constant for $E_{\text {tot }}>E_{\text {inc }}$, one gets

$$
\hat{p}(x ; t)=A(x) \mathrm{e}^{-c t} .
$$

Thus, in the $x-t$ space, the the state density has a product form.

Similar to the discussion in section 2 , this probability density is a density of the possible states of the stochastic process. The density of actually observed PD events is given by

$$
\hat{p}_{\mathrm{d}}(x, t)=c(x, t) \hat{p}(x ; t) .
$$

Recall that $c$ is assumed to be either zero or a constant. In regions where $c>0, \hat{p}_{\mathrm{d}}(x, t)$ differs from $\hat{p}(x, t)$ only by a proportionality factor. Thus

$$
\hat{p}_{\mathrm{d}}(x, t)=\hat{A}(x) \mathrm{e}^{-c t}
$$

within this region with a function $\hat{A}(x)$. Again a simple product form is obtained.

For summarizing we conclude that by a variable transformation $q \rightarrow x$ according to

$$
\begin{gathered}
E_{\mathrm{i}}= \pm E_{\mathrm{res}}-E_{0}-\frac{q}{\gamma}, \\
x=E_{\mathrm{i}} \mathrm{e}^{t / \tau},
\end{gathered}
$$

the density $p_{\mathrm{d}}(q, t)$ transforms into the density $\hat{p}_{\mathrm{d}}(x, t)$ which has a simple product form. The time dependence consists of an exponential decay $\mathrm{e}^{-c t}$ with an initial density $\hat{A}(x)$.

The same transformation can be performed on a measured PRPD pattern. Each PD event $(q, t)$ is represented by an event in $x-t$ space with the transformations equation (19). Thus, PRPD pattern is transformed into a point pattern in the $x-t$ space.

In figure 3 this transformation of the PRPD data is shown where the two steps $(q, t) \rightarrow\left(E_{\mathrm{i}}, t\right)$ and $\left(E_{\mathrm{i}}, t\right) \rightarrow(x, t)$ are shown separately.
The main idea of the parameter estimation algorithm is the following: The transformation $(q, t) \rightarrow(x, t)$ depends on the process parameter values. Only if the the parameter values are chosen correctly, the resulting transformed pattern has the simple structure of equation (18). The correct parameter values can be found by maximizing the compatibility of the transformed pattern with a probability density function as given in equation (18).

\subsection{A maximum-likelihood principle for parameter estimation}

A measure of the compatibility of a measured set of independent observations $x_{i}, i=1, \ldots, N$ with a given probability density $f_{\theta}(x)$, depending on some parameters $\theta=\left(\theta_{1}, \ldots, \theta_{\mathrm{p}}\right)$ is the likelihood

$$
L=\prod_{i=1}^{N} f_{\theta}\left(x_{i}\right)
$$

of the set of observations. A well-established method for parameter estimation is the maximum-likelihood (ML) method which consists in estimating the parameters $\theta$ by maximizing the likelihood with repect to $\theta$ :

$$
L(\theta)=\prod_{i=1}^{N} f_{\theta}\left(x_{i}\right)=\max .
$$

The new parameter estimation method for PRPD patterns adopts this principle in the following way: the parameter vector $\theta$ consists of the parameters $c, \tau, E_{0}$ and $E_{\text {res }}$ which are to be estimated from measured data. For given parameters $c, \tau, E_{0}$ and $E_{\text {res }}$, the measured PRPD pattern can transformed into the $x-t$ space by means of equation (19).

From this pattern in the $x-t$ space, the term $\hat{A}(x)$ is estimated by using equation (18) in the form

$$
\hat{A}(x)=\frac{\hat{p}_{\mathrm{d}}(x, t)}{\mathrm{e}^{-c t}}
$$

and integrating this equation over the time

$$
\hat{A}(x)=\frac{\int \hat{p}_{\mathrm{d}}(x, t) \mathrm{d} t}{\int \mathrm{e}^{-c t} \mathrm{~d} t}
$$

(see appendix B for details).

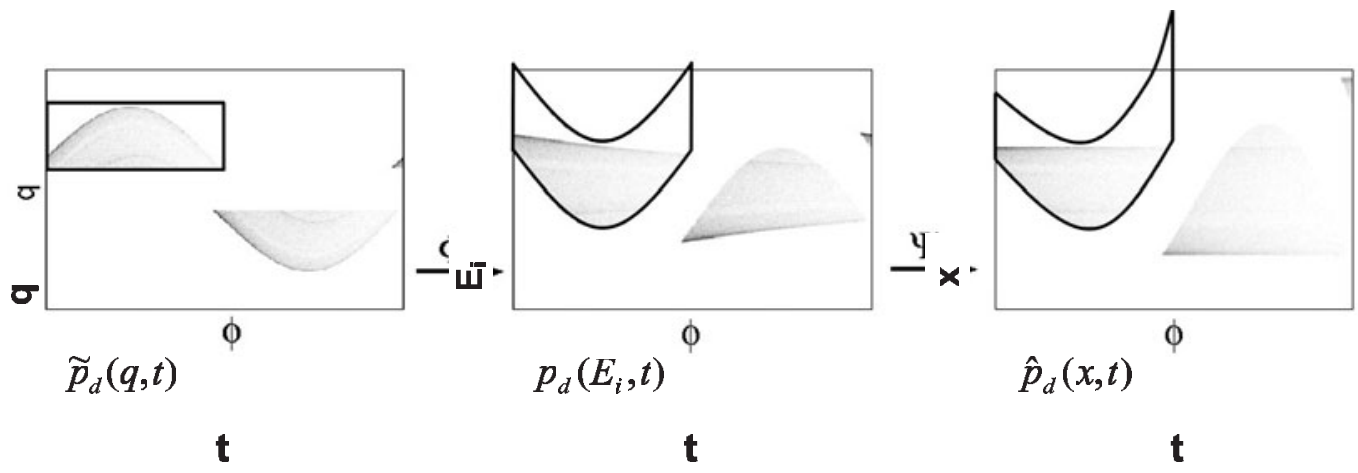

Figure 3. Transformation of PRPD data: $(q, t) \rightarrow(x, t)$. 


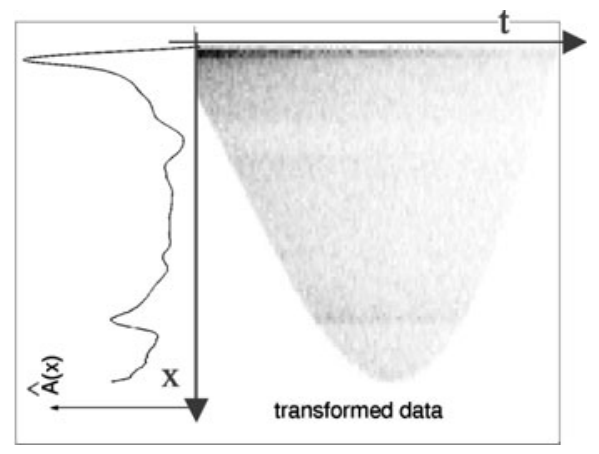

Figure 4. Estimation of $\hat{A}(x)$ from the transformed data.

The enumerator of equation (21) can be estimated from the measured data via a kernel estimator [32]. The denominator is known for specified model parameters. As an example, figure 4 shows the transformed data and an estimate of the initial distribution $\hat{A}(x)$.

The likelihood of the measured set of PD events is now calculated by treating each PD event as an independent realization of an underlying two-dimensional density function $\hat{p}_{\mathrm{d}}(x, t)$ :

$$
L\left(c, \tau, E_{0}, E_{\mathrm{res}}\right)=\prod_{i=1}^{N} \hat{p}_{\left(c, \tau, E_{0}, E_{\mathrm{res}}\right)}\left(x_{i}, t_{i}\right),
$$

where $\left(x_{i}, t_{i}\right), i=1, \ldots, N$, denote the single PD events in the $x-t$ space, and $\hat{p}_{\mathrm{d}}(x, t)$ is given by equation (18), using the estimate of $\hat{A}(x)$.

Maximizing this likelihood with respect to the process parameters leads to the estimates of the parameters.

Schematically the whole procedure is as follows:

1. transform data $\left(q_{i}, t_{i}\right) \rightarrow\left(x_{i}, t_{i}\right)$ (depending on $E_{\mathrm{res}}, E_{\mathrm{a}}$ and $\tau$ ),

2. estimate $\hat{A}(x)$,

3. $\hat{p}_{\mathrm{d}}(x, t)=\hat{A}(x) \mathrm{e}^{-c t}$,

4. $L\left(c, \tau, E_{0}, E_{\mathrm{res}}\right)=\prod_{i=1}^{N} \hat{p}_{\left(c, \tau, E_{0}, E_{\mathrm{res}}\right)}\left(x_{i}, t_{i}\right)$.

The parameters $E_{\text {res }}, E_{\mathrm{a}}$ and $\tau$ contribute to the likelihood function $L$ via the transformation $\left(q_{i}, t_{i}\right) \rightarrow\left(x_{i}, t_{i}\right)$ and the estimation of $A(x)$. The likelihood function is maximized by a standard optimization algorithm [33].

\subsection{Confidence intervals}

To obtain confidence intervals for the estimated parameter, a non-parametric bootstrap method is applied [34]. If the original data set $D$ consists of $N$ data points, $M$ new data sets, $D_{1}, \ldots, D_{M}$, each with $N$ data points are drawn from the original set with replacement. From each new data set parameters $\Theta_{i}^{*}$ are estimated. Those will be distributed around the original estimated parameters $\hat{\Theta}$ with a particular distribution. Confidence intervals can be obtained from the quantiles of that distribution [34].

\subsection{Discussion of the parameter estimation method}

In this section, some remarks on the parameter estimation method are made.
First, a remark on the statistical methodology must be made. The above-applied method for parameter estimation by maximizing $L\left(c, \tau, E_{0}, E_{\text {res }}\right)$ in equation (22) is, strictly speaking, not identical to the usual ML method, since for the density $\hat{p}_{\mathrm{d}}(x, t)$ the function $\hat{A}(x)$ is used which is estimated from the measured data. However, for large samples, the kernel estimator for $\hat{A}(x)$ yields the correct density. Thus, asymptotically, the likelihood is calculated correctly.

A second remark must be made on the estimation of the fields $\hat{E}_{0}, E_{\text {res }}$ and $E_{\text {inc. As }}$ As explained in section 3 , these fields cannot be determined in their absolute values (i.e. in units $\mathrm{V} \mathrm{m}^{-1}$ ). From a practical point of view, it would be desirable to get $\hat{E}_{0}$ and $E_{\text {res }}$ in units $E_{\text {inc }}$ which only requires the determination of the ratios of the fields.

With the parameter estimation method as described above, it is not possible to extract the value of $E_{\text {inc. This is due to }}$ the fact that the parameter estimation algorithm is based on data analysis in a region of the PRPD pattern where $c>0$. On the other hand, the inception field $E_{\text {inc }}$ is specified by the field value where $c$ drops down to zero. Since this is outside of the analysed region, the estimation algorithm cannot determine $E_{\text {inc }}$.

However, $E_{\text {inc }}$ can be calculated from the minimum measured charge $q_{\min }$. For simplification, we restrict the discussion to positive discharges. Setting $E_{\mathrm{tot}}=E_{\mathrm{i}}+E_{0}$ to its minimum value $E_{\mathrm{inc}}$ in equation (9), one gets

$$
q_{\mathrm{min}}=\gamma\left(E_{\mathrm{inc}}-E_{\mathrm{res}}\right) .
$$

Usually the minimum charge $q_{\min }$ can be determined quite well from the measured PRPD pattern. Once $E_{\text {res }}$ has been estimated by the parameter estimation algorithm, the field $E_{\text {inc }}$ can be calculated in the same units as the field $E_{\text {res }}$ by determining the minimum charge $q_{\mathrm{min}}$ from the measured PD pattern. With this information the fields $E_{0}$ and $E_{\text {res }}$ can be given in units of $E_{\text {inc }}$.

Thus, an additional input for the parameter estimation algorithm is the specification of $q_{\mathrm{min}}$. Note that this information is not necessary for the parameter estimation itself, but only for calculating the correct units for the estimated fields.

\subsection{Extension of the parameter estimation method to non-constant hazard rate}

The parameter estimation algorithm has been developed for the case of a constant first electron supply, i.e. a constant value of the parameter $c$. It can, however, be extended to the case of non-constant hazard rate as will be shown in the following.

For the case of a non-constant hazard rate which depends on the number of trapped charges and the applied external field, the parameter $c$ is a function of $E_{\mathrm{i}}$ and $E_{0}(t)$, or, equivalently, on $E_{\mathrm{i}}$ and $t: c=c\left(E_{\mathrm{i}} ; t\right)$. This means that the single parameter $c$ is replaced by a function which has many degress of freedom. For the following we assume that this function $c\left(E_{\mathrm{i}} ; t\right)$ is parametrized by some parameters $\psi=\left(\psi_{1}, \psi_{2}, \ldots\right)$. Instead of estimating one single value $c$, the task of the parameter estimation procedure is to estimate the parameter vector $\psi$, describing the mechanism of first electron supply.

For doing this, the above described parameter estimation method has to be modified only slightly. The transformation from the pattern $p\left(E_{\mathrm{i}} ; t\right)$ to the pattern $\hat{p}(x ; t)$ can be made 
exactly like for the case of constant $c$ since this transformation does not require $c$ to be constant.

A difference arises in equation (16) which is replaced by

$$
\hat{p}(x ; t)=A(x) \exp \left(-\int_{0}^{t} c\left(x, t^{\prime}\right) \mathrm{d} t^{\prime}\right),
$$

where the function $c\left(E_{\mathrm{i}} ; t\right)$ has been transformed into the function $c(x, t)$. The discharge event density $\hat{p}_{\mathrm{d}}(x, t)$ is then given by

$$
\hat{p}_{\mathrm{d}}(x, t)=c(x, t) A(x) \exp \left(-\int_{0}^{t} c\left(x, t^{\prime}\right) \mathrm{d} t^{\prime}\right),
$$

and the function $A(x)$ can be estimated using the equation

$$
A(x)=\frac{\int \hat{p}_{\mathrm{d}}(x, t) \mathrm{d} t}{\int c(x, t) \exp \left(-\int c(x, t) t^{\prime} \mathrm{d} t^{\prime}\right) \mathrm{d} t} .
$$

For given $c(x, t)$, or, equivalently, for a given parameter vector $\psi$, the function $A(x)$ can be estimated as before from the transformed pattern. The calculation of the likelihood can then be performed identically as for the case of constant $c$. The model parameters are estimated by maximizing the loglikelihood with respect to the model parameters $E_{\mathrm{res}}, E_{\mathrm{a}}, \tau$ and $\psi$.

Thus, the parameter estimation algorithm for non-constant hazard rate is identical to the case of constant hazard rate except that equations (18) and (21) have to be replaced by equations (25) and (26), and the single parameter $c$ is replaced by a set of parameters $\psi=\left(\psi_{1}, \psi_{2}, \ldots\right)$.

\section{The holographic property of the parameter estimation algorithm}

In the last sections a new algorithm for estimating parameters of a PD process has been presented. Basically, it consists of a ML approach which has to be applied to a contigueous region of the PD pattern for which $c>0$ is guaranteed. Such regions can be easily found in a measured PD pattern since $c$ must be positive in all regions where actually PD events are recorded. Aditionally, if positive charges are recorded at a point $(q, t)$, then $c>0$ for all point $\left(q^{\prime}, t\right)$ with $q^{\prime}>q$.

The simplest possibility of a contiguous region with $c>0$ is to take the whole positive or negative part of the pattern with exception of the horizontal zone around $q=0$ where no discharges are recorded ${ }^{3}$. In figure 5, this is displayed for positive discharges. Using this whole region ensures that all available data are taken into account for the parameter estimation procedure.

It must be stressed, however, that the analyis region can be chosen arbitrarily as soon as it does not contain regions with $c=0$. In figure 5, another example for a valid analysis region is shown in the lower part of the figure. The parameter estimation algorithm works equally well on this restricted region of the pattern because, in principle, the whole information about the process parameters is available within

\footnotetext{
3 The fact that no discharges are recorded in the zone around $q=0$ can be produced by two reasons: either there are no discharges with such small $q$ values. Then $c=0$ in this regions, or the threshold level of the PD measuring system prevents the recording of PD events with smaller charge.
}

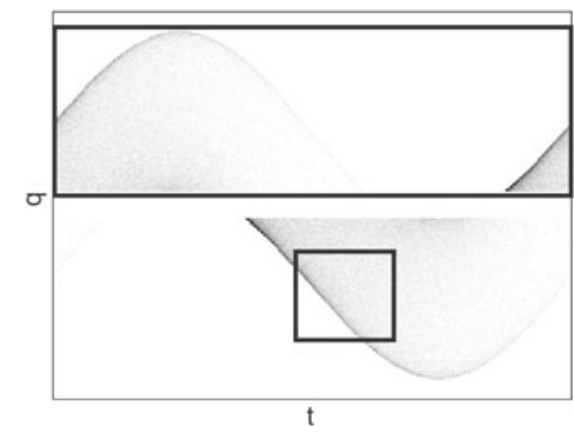

Figure 5. Two different contiguous regions in of the PRPD pattern where $c>0$ is given. Each of these regions can be used for performing a parameter estimation of the PD process.

arbitrarily small regions. This corresponds to the well-known fact that an experienced human PD specialist is able to analyse PD patterns even if not the whole pattern is available due to overlapping with other PD sources or noise. We call this phenomenon the holographic property of PRPD patterns.

On the other hand, of course, due to statistical reasons, the accuracy of the parameter estimation is reduced if only a part of the pattern is used for the parameter estimation.

\section{Application to simulated data}

Numerous sets of artificial data were produced by simulations to investigate the accuracy of the algorithm. Figure 6 shows four PRPD patterns from simulations with different parameter combinations. $E_{0}$ and $E_{\text {res }}$ are kept fixed at values of 5 and 0.3 respectively. The important parameters $\tau$ and $c$ are varied. A discharge in a spherical void under sinusoidal voltage of $50 \mathrm{~Hz}$ was assumed. The simulation time was set to $20 \mathrm{~min}$. For the parameter estimation, the whole upper half of the pattern was used with exception of the the zone stripe $q=0$ where no discharges are recorded.

Figure $6(a)-(d)$ show the data from the simulations. These particular combinations of $c$ and $\tau$ are chosen to trace out the limits of the parameter estimation algorithm.

Table 1 shows estimates for the different parameters and their confidence limits. Note that the confidence intervals are sometimes asymmetric. In most of the cases an estimation is possible, if not-e.g. the estimation for $\tau$ in $(a)$ and $(d)$ it reflects the fact that this discharge parameter has little or no influence on the pattern as discussed in section 3.1. This lack of information in the data is reflected in large confidence intervals.

In the following, the four examples are discussed briefly.

(a) Parameter $\tau$ was chosen so that the internal field decays very slowly. There is a fast discharge about every fifth period. During that time the internal field declines only by $1 \%$ due to drift processes. Thus the drift processes have negligible influence to the process dynamics which is governed by the fast discharges. As a result, $\tau$ cannot be estimated accurately. However, a lower bound for $\tau$ is found by the algorithm.

(b) With this parameter setting, the internal field decays during ten periods to a value of $1 / \mathrm{e}$ and there is a discharge approximately every tenth period. Both $c$ and $\tau$ can be estimated accurately. 

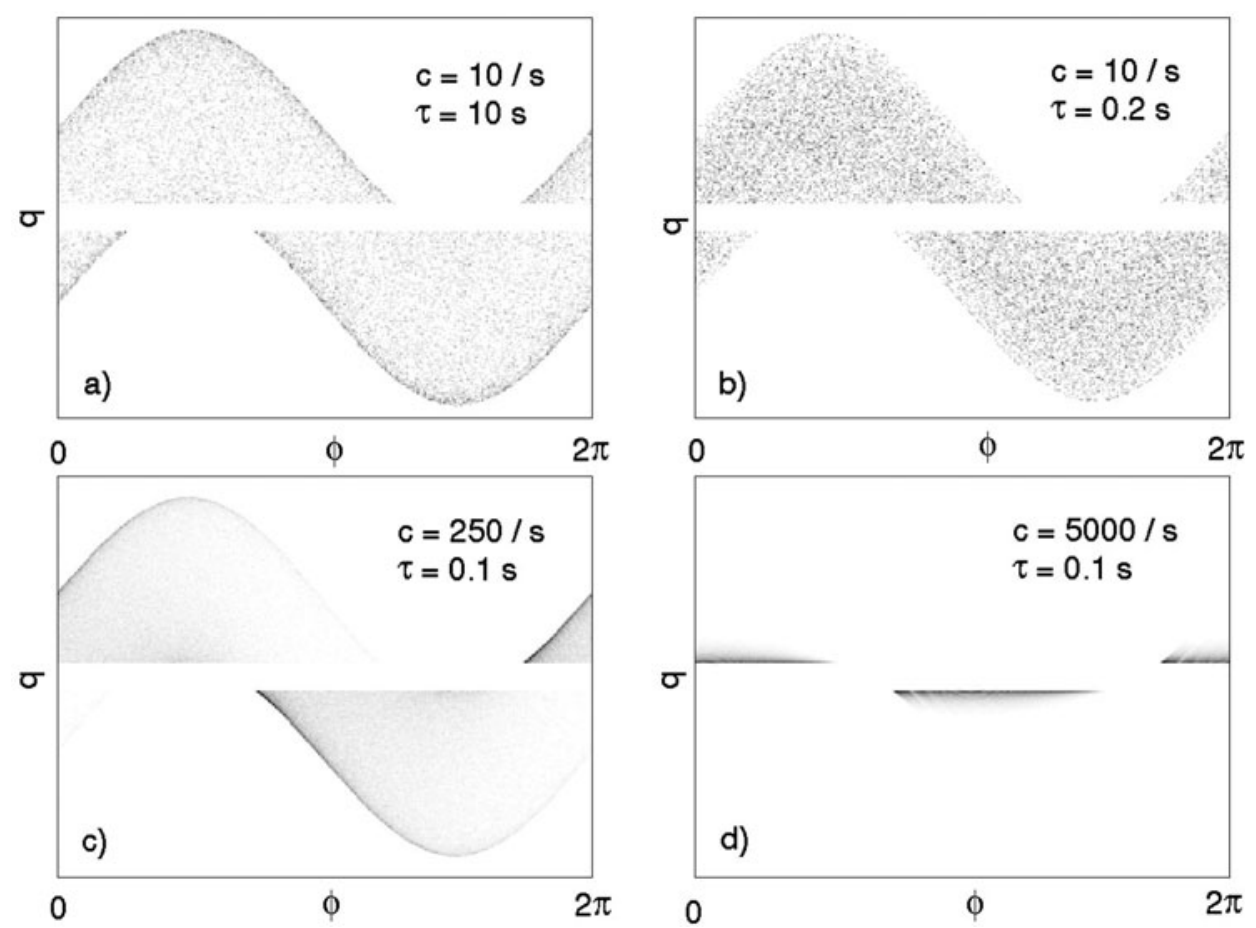

Figure 6. Simulated data for different parameter combinations of $\tau$ and $c$.

Table 1. True values and estimates of parameters $c, \tau, E_{0}$ and $E_{\text {res }}$ for simulated data.

\begin{tabular}{lrllllllr}
\hline Figure & $\begin{array}{l}c \\
\left(\mathrm{~s}^{-1}\right)\end{array}$ & $\begin{array}{l}c_{\text {est }} \\
\left(\mathrm{s}^{-1}\right)\end{array}$ & $\begin{array}{l}\tau \\
(\mathrm{s})\end{array}$ & $\begin{array}{l}\tau \text { (estim. }) \\
(\mathrm{s})\end{array}$ & $\begin{array}{l}\hat{E}_{0} \\
\left(E_{\text {inc }}\right)\end{array}$ & $\begin{array}{l}\hat{E}_{0}(\text { estim. }) \\
\left(E_{\text {inc }}\right)\end{array}$ & $\begin{array}{l}E_{\text {res }} \\
\left(E_{\text {inc }}\right)\end{array}$ & $\begin{array}{l}E_{\text {res }}(\text { estim. }) \\
\left(E_{\text {inc }}\right)\end{array}$ \\
\hline$(a)$ & 10 & $13.6 \pm 4.5$ & 10 & $140_{-135}^{+\infty}$ & 5 & $5.01 \pm 0.02$ & 0.3 & $0.29 \pm 0.02$ \\
$(b)$ & 10 & $13.18 \pm 4.7$ & 0.2 & $0.22 \pm 0.03$ & 5 & $4.97_{-0.1}^{+0.04}$ & 0.3 & $0.3 \pm 0.01$ \\
$(c)$ & 250 & $249.5 \pm 1.5$ & 0.1 & $0.098 \pm 0.003$ & 5 & $5.01 \pm 0.03$ & 0.3 & $0.3 \pm 0.01$ \\
$(d)$ & 5000 & $4950 \pm 30$ & 0.1 & $0.2_{-0.15}^{+\infty}$ & 5 & $6 \pm 3$ & 0.3 & $0.2 \pm 0.2$ \\
\hline
\end{tabular}

(c) $\tau$ is similar to the value in $(b)$. The internal field decays during five periods to a value of $1 / \mathrm{e}-c$ is higher. The parameters can be estimated with high precision.

(d) $c$ is extremely high. As soon as the total field exceeds the inception field, a discharge takes place. Drift processes have no influence to the process dynamics. Thus $\tau$ cannot be estimated.

In all cases, the values of $\hat{E}_{0}$ and $E_{\text {res }}$ could be determined with a high accuracy.

As an example of the holographic property, in figure 7 the estimated parameter values for two different analysis regions are shown. It can be seen that the parameter estimation works well for both regions. However, the smaller the region, the larger are the error bounds because fewer data enter the analyis.

\section{Application to measured data}

The algorithm was applied to data obtained from an artificial spherical void in epoxy resin under sinusoidal voltage. Seventy-two measurement were made during $24 \mathrm{~h}$ (20 min per measurement) at constant voltage level [35]. The PRPD patterns are provided as a $256 \times 256$ numerical array where each bin is specified by its phase position and the charge amplitude

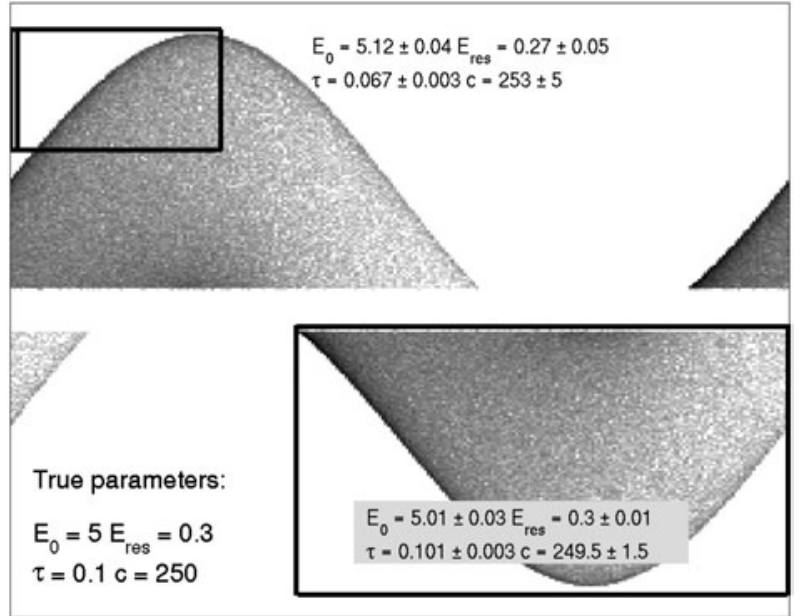

Figure 7. Holographic property.

and contains the number of recorded PD events for this phase position and charge. In [36], the details on the experimental setup and the measurement method can be found. Figure 8 shows a selection of these measurements.

The PRPD patterns can be divided into two groups. Measurements 1-22 and 49-72 (group I) show nearly identical 
patterns; so do measurements 26-45 (group II). Measurements 23-25 and 46-48 show the change of the two pattern types into one another. The two patterns correspond to the daynight cycle. At daytime (group I), more first electrons are available due to UV radiation of the sunlight. At night, few first electrons are present, leading to patterns with a small number of discharges.

The patterns show a strong asymmetry, suggesting a significant difference in the discharge behaviour for positive and negative discharges. Therefore, the parameter estimation is done separately for the two half-waves. The parameter estimation region was chosen like indicated in figure 8. Only the first part of the pattern has been used.

\subsection{Estimation of the process parameters}

Figure 9 shows the estimates of $c$ for the different measurements for positive and negative discharges. The change of $c$ from measurements of group I to those of group II is enormous. It changes by the factor of about 100. A small $c$ means a lack of first electrons while a large $c$ corresponds to an abundance of first electrons.

It can be seen that the estimation of the parameter $c$ is possible within reasonable error bounds.

This is different for the parameter $\tau$ which describes the decay of the deployed charge by drift and recombination processes. In figure 10, the $\tau$ estimation for the positive
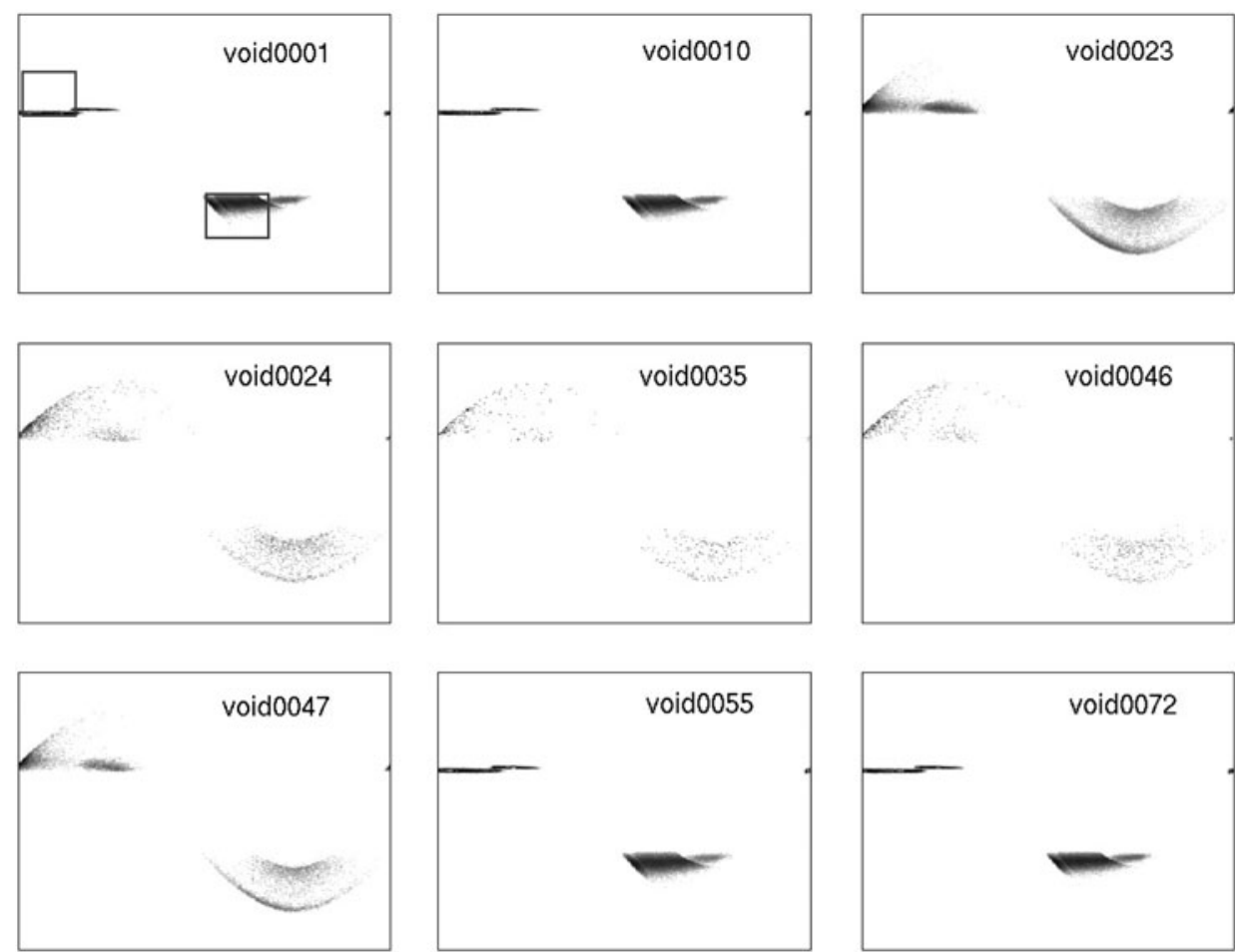

Figure 8. Selection of measured data. The upper-left pattern the chosen analysis region is indicated. The process parameters have been estimated from the data within this region.
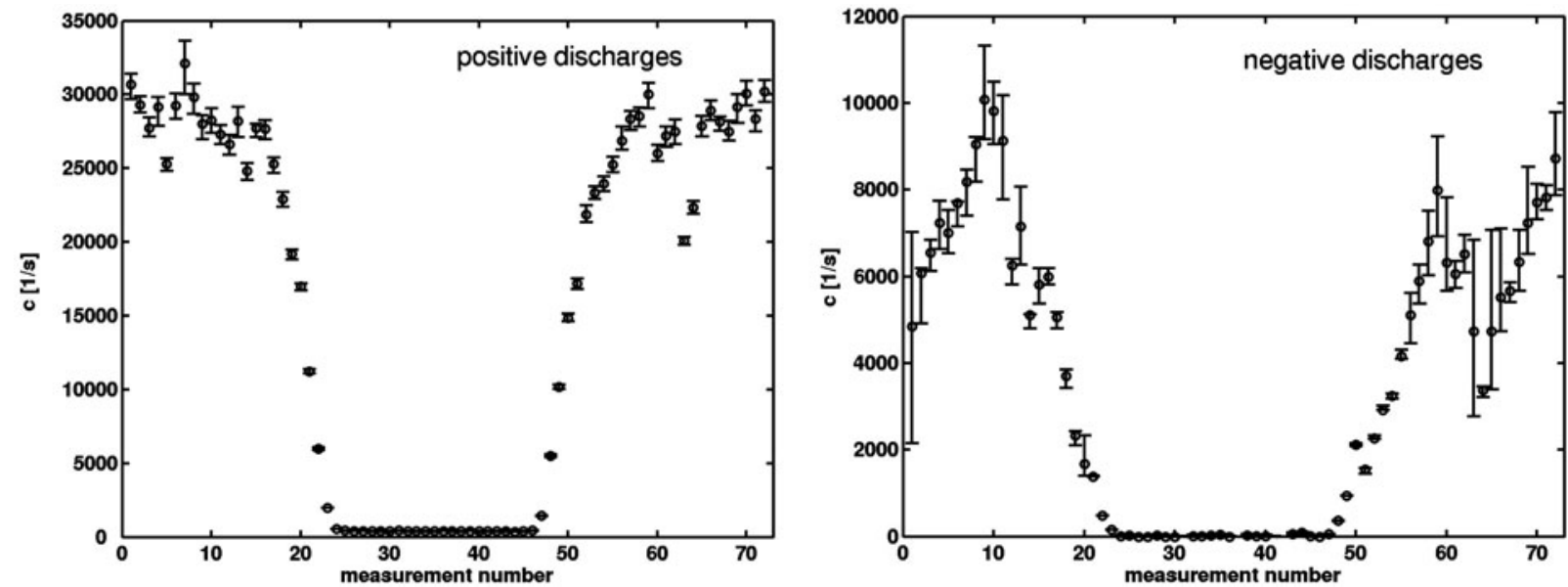

Figure 9. Estimation of parameter $c$ for positive and negative discharges. The circles show the estimated value, the vertical bars indicate the $90 \%$ confidence interval. 
discharges is shown. For negative discharges, similar results are obtained. The estimated value for $\tau$ is $3-6 \mathrm{~s}$ for most cases; however, the analysis of the confidence interval shows that, actually, the parameter is not identifiable. In all cases, the upper limit of the confidence interval is $\infty$, indicating that the exact value cannot be determined. The reason for this non-identifiability is that $\tau$ is much larger than the mean time interval between successive discharges.

However, in each case a lower bound is found in the range of 2-4 s. A $\tau$ value below this lower bound would not be compatible with the measured data.

The estimation of the parameter $\hat{E}_{0}$ is shown in figure 11 . For patterns of group I, the error bars are quite large. The reason for this behaviour is that the patterns of group I are nearly degenerate, consisting of a rather narrow stripe. The fit of the partial differential equation in this small region is much more difficult as for the large regions which are available

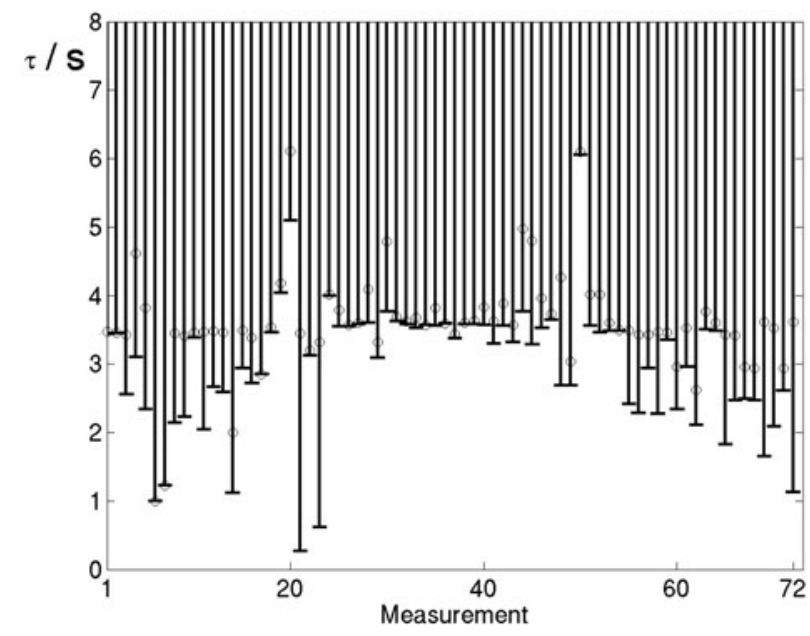

Figure 10. Estimation of parameter $\tau$ for positive discharges. The circles show the estimated value, the vertical bars indicate the $90 \%$ confidence interval. In each case, the upper limit of the confidence interval is $\infty$.

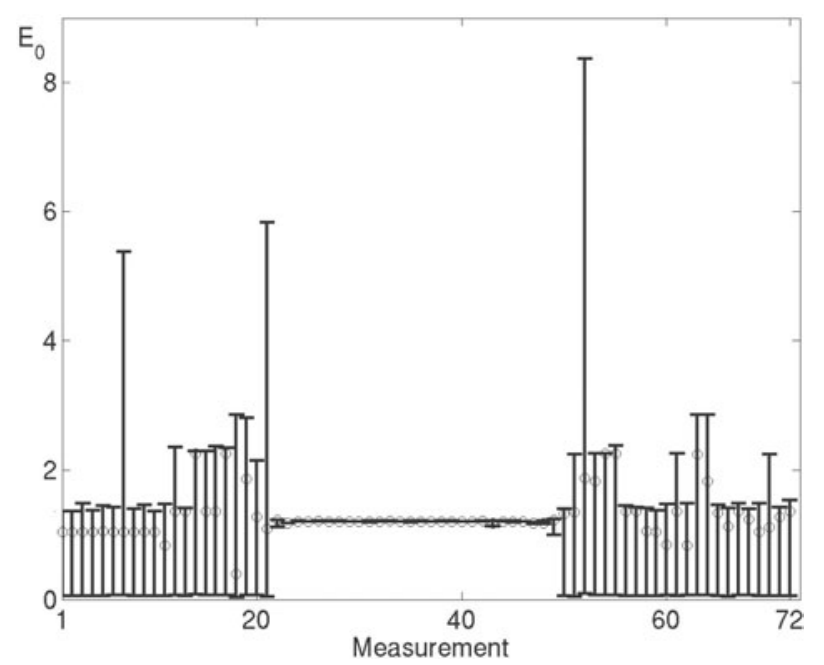

Figure 11. Estimation of parameter $\hat{E}_{0}$ for positive discharges. The circles show the estimated value and the vertical bars indicate the $90 \%$ confidence interval. for patterns of group II. Consequently, the error bounds for patterns of group II are much smaller.

Note that the estimation only takes into account the internal structure of the PRPD pattern as described by the partial differential equation. In practice, the PRPD inception voltage can be measured easily. Usually, $\hat{E}_{0}$ is obtained by determination of the inception voltage which corresponds to $\hat{E}_{0}=1$ in units of $E_{\text {inc. }}$. The presented parameter estimation algorithm can be viewed as a complementary way of determination of $\hat{E}_{0}$.

For the parameter estimation algorithm, no boundary condition for $\hat{E}_{0}$ has been set. Thus, the estimation algorithm also accepts values below 1 which, physically, is not realistic, since for $\hat{E}_{0}<E_{\text {inc }}$, no discharge activity can take place. The inclusion of such physical boundary conditions into the statistical parameter estimation procedure will further improve the results.

In figure 12, the estimation of $E_{\text {res }}$ is shown. For measurements of group II, the inception field can be estimated quite well. It has a very small value, slightly above zero. Similar to $\hat{E}_{0}$, this parameter can only be estimated with large error bounds for measurements of group I. Similarly to the $\hat{E}_{0}$ estimation, in these cases also unphysical values larger than unity are given. Again, including the physical restriction $E_{\mathrm{res}}<E_{\mathrm{inc}}$ would further improve the quality of the results.

\subsection{Comparison of simulated data with measured patterns}

The estimated process parameters were used for simulating PRPD patterns. If the parameters are estimated correctly, the simulated pattern should have a high similarity with the measured pattern. In figure 13 two examples of simulated patterns are shown together with their measured counterpart. In table 2 the statistical parameters of the measured and simulated patterns are shown. Mean, variance, skewness and kurtosis of the one-dimensional densities were evaluated from the patterns, separately for the positive and negative charges. Mean and variance are given in units bins (note that the patterns are provided as a $256 \times 256$ array).

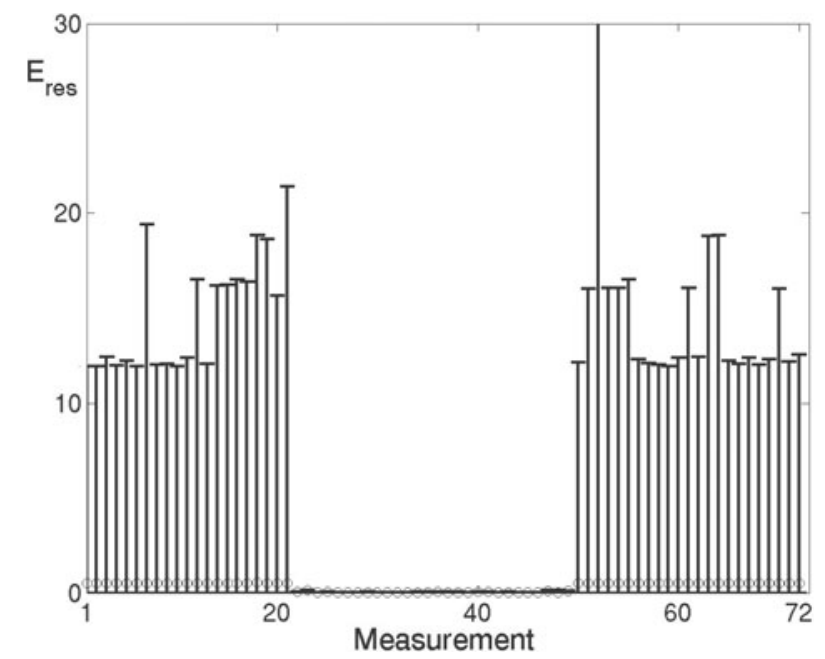

Figure 12. Estimation of parameter $E_{\text {res }}$ for positive discharges. The circles show the estimated value and the vertical bars indicate the $90 \%$ confidence interval. 

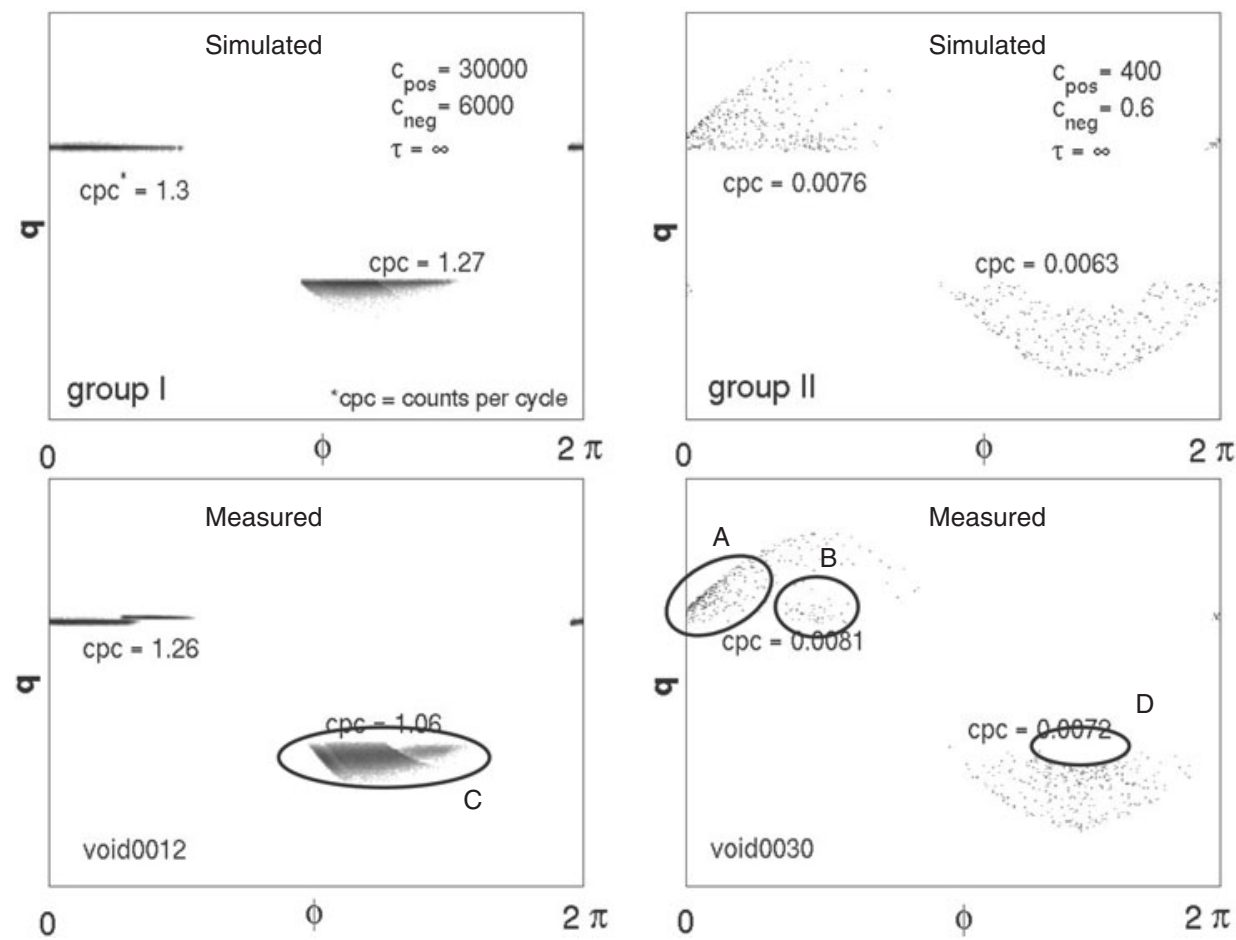

Figure 13. Simulated data with parameter sets from estimation in comparison with corresponding measured patterns.

Table 2. Statistical parameters of the measured and re-simulated patterns shown in figure 13.

\begin{tabular}{|c|c|c|c|c|c|c|c|c|}
\hline & \multicolumn{2}{|c|}{ Mean } & \multicolumn{2}{|c|}{ Variance } & \multicolumn{2}{|c|}{ Skewness } & \multicolumn{2}{|c|}{ Kurtosis } \\
\hline & Meas. & Simul. & Meas. & Simul. & Meas. & Simul. & Meas. & Simul. \\
\hline \multicolumn{9}{|c|}{ Void0021 } \\
\hline$q_{\mathrm{pos}}$ & 38.571 & 43.646 & 1.976 & 0.976 & 0.872 & 0.074 & -0.242 & 0.06 \\
\hline$t_{\mathrm{pos}}$ & 24.793 & 22.097 & 306.797 & 228.514 & 0.460 & 0.371 & -0.728 & -0.661 \\
\hline$q_{\text {neg }}$ & -46.836 & -44.395 & 14.581 & 2.962 & -0.345 & -1.292 & 0.011 & 3.375 \\
\hline$t_{\text {neg }}$ & 153.26 & 150.208 & 191.491 & 213.61 & 0.516 & 0.449 & -0.188 & -0.547 \\
\hline \multicolumn{9}{|c|}{ Void0030 } \\
\hline$q_{\mathrm{pos}}$ & 57.558 & 57.965 & 208.02 & 185.375 & 0.810 & 0.967 & -0.273 & -0.081 \\
\hline$t_{\mathrm{pos}}$ & 30.762 & 34.144 & 624.147 & 621.526 & 0.948 & 0.466 & 0.119 & -0.546 \\
\hline$q_{\text {neg }}$ & -65.409 & -65.167 & 153.835 & 220.135 & -0.500 & -0.353 & -0.792 & -1.059 \\
\hline$t_{\text {neg }}$ & 189.86 & 195.458 & 498.107 & 1085.64 & -0.064 & -0.106 & -0.358 & -0.918 \\
\hline
\end{tabular}

It can be seen that the simulated patterns show a high similarity with the measured patterns. Typical pattern features are reproduced correctly. Examples are:

- the increased point density at the upper edge of the pattern, especially at $\varphi \approx 0$ (region A in figure 13);

- the occurrence of a second point accumulation for small positive discharges at $\varphi \approx \pi / 2$ (region B in figure 13);

- the occurrence of two adjoining point clowds for negative discharges (region $\mathrm{C}$ in figure 13). In the simulated pattern, the separation between these two clouds is less pronounced than in the measured pattern, but it is clearly present.

- the lack of discharges in region D in figure 13. This lack is only present for negative discharges.

Note that the different pattern structure for positive and negative discharges is reproduced correctly. This is a very strong result due to the following reason. For the parameter estimation the two half-waves have been analysed separately, leading to different PD parameters for positive and negative discharges. No explicit mutual coupling between the two discharge polarities have been taken into account. The only information which is exploited for the parameter estimation is the internal structure of the point pattern in a predefined region of the PRPD pattern.

In reality, however, the positive and negative discharges are coupled. The discharge behaviour in the positive half-wave has direct consequences for the discharge behaviour in the negative half-wave, and vice versa. This mutual coupling is not used for the parameter estimation. However, when simulating patterns with the estimated parameter values, this coupling is taken into account. For patterns with a difference between negative and positive discharges it is therefore far from being an automatism that simulation of the PD process (with separately estimated parameters) lead to PRPD patterns with the correct structure.

In figure 13 , the counts per cycle for positive and negative discharges are displayed for the measured patterns as well as 
for the simulated ones. The simulations have a slightly higher number of discharges than the measured patterns. The reason for this systematic deviation is not yet clear. A possible reason could be the dead times of the PD measuring system leading to a number of discharges which is systematically too low. This assumption is supported by the observation that the deviation is larger for pattern with a high values of counts per cycle.

It must be stressed that the values of counts per cycle for the measured patterns are not an input of the parameter estimation algorithm. The parameter estimation is based on the master (equation (13) or (16)) which is is a differential equation for the point density which is independent on the absolute pattern density. Consequently, the estimation of the parameters is based solely on the relative temporal change of the point density within the pattern, not on the absolute value of the point density. Taking this into account, the reproduction of the point density (measured by the counts per cycle) within an error range of only some $10 \%$ is a validation of the model and the presented parameter estimation procedure.

However, some properties of the pattern are not reproduced by the simulation. For example, the step in the horizontal stripe of figure 13 does not appear in the simulation. Instead, the simulation yields a horizontal stripe without a charge step.

Regarding the statistical parameters in table 2, there are some significant differences between the original and the simulated patterns, especially for the pattern of group I (void0012, left patterns in figure 13). For example, the variance of the negative charge distribution for void0012 is much too low in the simulated pattern. For skewness and kurtosis occasionally large deviations arise.

The reason for these deviations probably is originated in too simplified a model for the discharge process. Note that the description of the discharge process with only two physical parameters $c$ and $\tau$ (the other parameters $E_{0}$ and $E_{\text {res }}$ are, striktly speaking, not discharge parameters but instead describe the external field) is a very crude approximation to the true complexity of the physical processes involved. For example, the physical model for void discharges developed in $[5,26]$ is much more complicated than the used simple model of [8]. Additionally, in the used parameter extraction algorithm, the first electron supply has been assumed to be constant ignoring first electron supply by surface electrons. Therefore, is expected that the model cannot capture all details of the pattern.

In contrast, actually it is very surprising that the underlying simple model is able to describe so much detail of the measured PRPD pattern, and that the parameter estimation procedure based on this model works that well. In spite of its simplicity, the model seems to describe correctly the main features of the physical process for the examined data.

\section{Discussion}

This section contains some additional remarks on the presented parameter estimation algorithm.

The approach allows one to extract information about the physical parameters of a PD without a simulation of the discharge process. To the knowlegde of the authors, there is no similar approach available in literature. Usually, the physical parameters of a PD process are estimated by simulation of the process according to a physical model, comparing the output of the simulation with measured data, and changing the parameters until a match between simulated and measured data is obtained. The present method, in contrast, is able to directly estimate the physical process parameters by data analysis. Moreover, error bounds can be given easily, which is very difficult with a trial-and-error parameter estimation method using simulation.

Compared with other data analyis methods used, like the extraction of statistical properties such as mean, skewness, etc, from measured PRPD pattern, the proposed method is superior since it renders physical information about the PD process which describes the nature of the process much better than in terms of simple descriptive statistical numbers. As has been shown in section 6 , a change of one of the physical parameters (availability of first electrons) leads to patterns with a completely different structure. The analysis of the physical parameters shows clearly that only this one parameter has changed while the others remain constant. Another example is the comparison of patterns of the same PD defect at different applied voltages. Here the usual statistical measures are different. However, the physics of the discharge process, described by the parameters $c$ and $\tau$, remains the same, which can be clearly revealed by the proposed method.

It must be stressed that, as for all physical models, the parameter estimates only make sense if the model for the discharge process is correct. The presented algorithm is based on a fairly simple model with only few physical parameters. As mentioned above, it is to be expected that there may be situations where the model may be too simplified and thus inappropriate. For example, effects like shielding due to conductivity of cavity walls or multi-PD phenomena in the same cavity cannot be described. The great advantage of the model developed in [8] is that it allows a direct parameter estimation as described in this paper. This is in contrast to more detailed physical models like those used in $[5,26]$ which describe the process more accurately but render no possibility to directly extract the parameter values from measured data. Thus, the authors hope that the used simplified model together with the possibility to extract the parameters directly from the data helps to get a better understanding of measured PD phenomena, even if the model can only be an approximative one. Note, however, that the aim of this paper is not to prove the validity of the underlying model assumption but to describe an approach for physical parameter extraction based on the stochastic model description of [8].

The presented algorithm does not make use of the absolute charge value but is based on the temporal change of the pattern as expressed in equation (6). This independence of the absolute charge means that, for the parameter estimation, the electromagnetic coupling between PD source and PD sensor need not be known. This is very advantageous in practice where the position of the PD source often is not known exactly.

The holographic property of the PRPD pattern is fully exploited by the presented parameter estimation. For the parameter estimation, it is not necessary to use the whole pattern but a restriction to a part of the pattern is possible. This large freedom in choosing the analysis region has a huge impact on the practical usefulness of the approach. In practice, 
measured PDs are often corrupted by noise sources, or many PD sources are active at the same time, leading to overlapping patterns. In these cases, traditional PD analysis methods fail. For example, deriving statistical parameters like skewness or kurtosis makes no sense if more than one PD source is active, or if a strong noise source is present. Our approach, in contrast, can easily deal with noise and multi-source patterns as long as there are regions in the pattern which can be assigned to a single defect. It is possible to restrict the parameter estimation to exactly this region and extract information on this single PD defect.

The parameter estimation method has been presented for the case of void discharges with a constant supply of first electrons. It has been shown in section 3.6 that the approach can be extended to the case of first electron supply by trapped surface charges. In principle, the same idea of parameter estimation could also be applied to other kinds of PD sources as well, e.g. corona discharges or surface discharges. This is possible since these discharges are described by the same kind of master equation [8]. The only difference is the relation between the field change $\Delta E$ and the measured charge $q$ of the discharge which is linear for void discharges and takes the form of a power law for other discharges [8]:

$$
q=-\gamma(\Delta E)^{\alpha} \text {. }
$$

An adoption of the presented method to other discharge types would therefore be possible. Moreover, the exponent $\alpha$ could be treated as an additional parameter which could be estimated analogously to the others. The resulting algorithm would then not only yield the physical parameters as described above, but also additional information on the type of the discharge. This could be used as a means for classification of PD sources based on physical parameters.

\section{Conclusion}

In this paper, a novel method for estimation of physical discharge parameters for PDs in voids has been presented. The method is based on a recently developed framework for modelling a PD process as a stochastic process governed by a master equation.

The parameter estimation algorithm makes use of the specific form of this master equation. By using a ML approach, the parameters can be estimated. Furthermore, error bounds for the parameter estimates can be given. With this method a compact description of a PD pattern in terms of the physical parameters governing the discharge process is possible. In contrast to purely descriptive statistical parameters, the physical parameters can be interpreted in terms of discharge physics.

The method has been tested by application to different simulated PRPD patterns. It has also been successfully applied to measured data of a series of PRPD measurements of a void in epoxy resin subjected to varying UV radiation. The change of first electron availability is reflected by a clear change of the corresponding physical parameter $c$.

The parameter estimation method works on a contiguous region of the PRPD pattern which may be only a small part of the actually measured pattern. This holographic property of the method allows the application of the method even on patterns with are strongly corrupted by noise, as long as there are regions in the $q-t$ space which are essentially free of noise. For patterns with several active PD defects, the single defects can be analysed separately as long as they are separated, at least partly, within the pattern. This is not possible with usual pattern analysis techniques.

\section{Acknowledgment}

We are very grateful to Power Diagnostics $\mathrm{GmbH}$ for their permission to use their data for testing the parameter extraction method.

\section{Appendix A. Transformation from $\left(E_{\mathrm{i}} ; t\right)$ to $(x, t)$}

For regions $\left\{\left(E_{\mathrm{i}} ; t\right) \mid c\left(E_{\mathrm{i}} ; t\right)>0\right\}$ and under the assumption that $f$ has the form given in equation (5), the master equation describing the process dynamics has the form (see equation (13)):

$\frac{\partial}{\partial t} p\left(E_{\mathrm{i}} ; t\right)=\left(\frac{1}{\tau}-c\right) p\left(E_{\mathrm{i}} ; t\right)+\frac{E_{\mathrm{i}}}{\tau} \frac{\partial}{\partial E_{\mathrm{i}}}\left(p\left(E_{\mathrm{i}} ; t\right)\right)$.

We consider the transformation $E_{\mathrm{i}} \rightarrow x=E_{\mathrm{i}} \mathrm{e}^{t / \tau}$.

Normalization of the probability density has to be fulfilled at every time $t$, thus we have

$$
\hat{p}(x ; t)=p\left(E_{\mathrm{i}}(x) ; t\right) \cdot \frac{\mathrm{d} E_{\mathrm{i}}}{\mathrm{d} x}=p\left(E_{\mathrm{i}} ; t\right) \mathrm{e}^{-t / \tau} .
$$

The temporal derivative of $\hat{p}(x ; t)$ is given by

$$
\frac{\mathrm{d} \hat{p}(x ; t)}{\mathrm{d} t}=\frac{\partial \hat{p}}{\partial x} \frac{\partial x}{\partial t}+\frac{\partial \hat{p}}{\partial t}=\frac{x}{\tau} \frac{\partial \hat{p}}{\partial x}+\frac{\partial \hat{p}}{\partial t}
$$

The LHS of equation (27) now reads

$\frac{\mathrm{d}}{\mathrm{d} t} p\left(E_{\mathrm{i}} ; t\right)=\frac{\mathrm{d}}{\mathrm{d} t}\left(\hat{p}(x ; t) \mathrm{e}^{t / \tau}\right)=\mathrm{e}^{t / \tau}\left(\frac{x}{\tau} \frac{\partial \hat{p}}{\partial x}+\frac{\partial \hat{p}}{\partial t}\right)+\frac{\hat{p}}{\tau} \mathrm{e}^{t / \tau}$.

With

$$
\frac{\mathrm{d}}{\mathrm{d} E_{\mathrm{i}}}=\frac{\mathrm{d} x}{\mathrm{~d} E_{\mathrm{i}}} \frac{\mathrm{d}}{\mathrm{d} x}=\mathrm{e}^{t / \tau} \frac{\mathrm{d}}{\mathrm{d} x},
$$

the RHS of equation (27) takes the form

$$
\left(\frac{1}{\tau}-c\right) \hat{p}(x ; t) \mathrm{e}^{t / \tau}+\frac{x \mathrm{e}^{-t / \tau}}{\tau} \mathrm{e}^{t / \tau} \frac{\mathrm{d}}{\mathrm{d} x} \hat{p}(x ; t) \mathrm{e}^{t / \tau} .
$$

Comparing LHS and RHS, most of the terms cancel out, leading to

$$
\frac{\partial p(\hat{x} ; t)}{\partial t}=-c(x, t) \hat{p}(x ; t) .
$$

\section{Appendix B. Estimation of $A(x)$}

Given a transformed PRPD pattern in the $x-t$ space, each measured PD event corresponds to one point of this pattern. From theory (see appendix A) it is known that the process has the probability density

$$
\hat{p}(x ; t)=A(x) \mathrm{e}^{-c t},
$$


where $A(x)$ is an initial distribution which is not known but must be estimated from the data. The above equation is valid only for a contiguous region for which $c>0$ (see section 3.2). While this region has generally a simple form in the $q-t$ space of the original pattern, it can have a complicated form in the $x-t$ space (see figure 14).

The measured PD pattern in the $x-t$ space has a point density which is given by

$$
\hat{p}_{\mathrm{d}}(x, t)=c(x, t) \hat{p}(x, t) .
$$

The task is to estimate the distribution $A(x)$ such that equation (30) is consistent with the measured data.

By integrating $\hat{p}_{\mathrm{d}}(x, t)$ over $t$ one gets (see figure 14)

$$
\begin{gathered}
\int_{0}^{T} \hat{p}_{\mathrm{d}}(x, t) \mathrm{d} t=\int_{a}^{b} c \hat{p}(x ; t) \mathrm{d} t \\
=c A(x) \int_{a}^{b} \mathrm{e}^{-c t} \mathrm{~d} t \\
=A(x)\left(\mathrm{e}^{-c b}-\mathrm{e}^{-c a}\right),
\end{gathered}
$$

where $a$ and $b$ are the interval boundaries for the region where $c>0$. Thus

$$
A(x)=\frac{\int_{0}^{T} \hat{p}_{\mathrm{d}}(x, t) \mathrm{d} t}{\mathrm{e}^{-c b}-\mathrm{e}^{-c a}} .
$$

Thus, in principle, the density $A(x)$ can be estimated by summing all PD events along a horizontal line in the $x-t$ space and dividing by the factor $\left(\mathrm{e}^{-c b}-\mathrm{e}^{-c a}\right)$. In practice, however, the resulting density $A_{\text {est }}(x)$ is very peaked, especially when the pattern consists of few points. Therefore, the calculated estimated density is additionally smoothed with a smoothing kernel. The resulting estimator for the initial distribution $A(x)$ can thus be interpreted as a kernel estimator.

In order to yield a valid probability density, the resulting estimated density

$$
\hat{p}_{\text {est }}(x, t)=A_{\text {est }}(x) \mathrm{e}^{-c t}
$$

is normalized in order to get

$$
\int_{t} \int_{x} \hat{p}_{\text {est }}(x, t) \mathrm{d} x \mathrm{~d} t=1,
$$

where the integration extends over the whole considered region of the $x-t$ space.

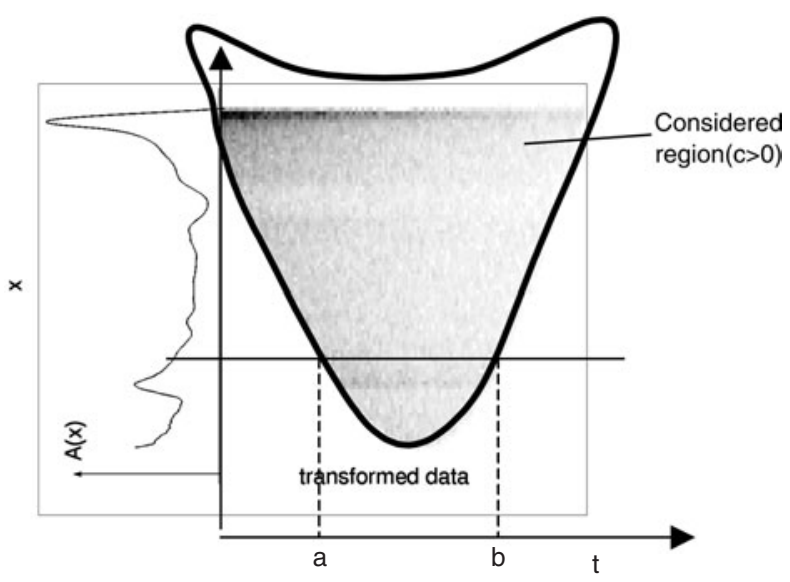

Figure 14. Estimation of the initial distribution $A(x)$ from the data. For a given $\mathrm{x}$, the value of the initial distribution $A(x)$ can be derived from the data by integrating all PD events along a horizontal line.

\section{References}

[1] Devins J C 1984 The physics of partial discharges in solid dielectrics IEEE Trans. Electric. Insul. 19 475-95

[2] van Brunt R J and Kulkarni S V 1990 Stochastic properties of Trichel-pulse corona: a non-Markovian random point process Phys. Rev. A 42 4908-32

[3] van Brunt R J 1991 Stochastic properties of partial-discharge phenomena IEEE Trans. Electric. Insul. 26 902-48

[4] Fromm U and Gulski E 1994 Statistical behaviour of internal partial discharges at DC voltage Prod. 4th Intern. Conf. on Properties and Applications of Dielectric Materials (Brisbane, Australia, 1994) paper 6230

[5] Niemeyer L 1995 A generalized approach to partial discharge modeling IEEE Trans. Diel. Electric Insul. 2 510-28

[6] Hoof M and Patsch R 1997 A physical model: describing the nature of partial discharge pulse sequence ICPADM 97 (Seoul, Korea 1997) pp 283-6

[7] Patsch R and Hoof M 1998 Physical modelling of partial discharge patterns IEEE 6th Intern. Conf. on Conduction and Breakdown in Solids ICSD'98 (Västeras Sweden) 114-8

[8] Heitz C 1999 A generalized model for partial discharge processes based on a stochastic process approach J. Phys. D 32 1012-23

[9] Okamoto T, Kaot T, Yokomizu Y and Suzuoki Y 2001 PD charactaristics as a stochastic process and its integral equation under sinusoidal voltage IEEE Trans. Diel. Electric. Insul. 8 82-90

[10] 1969 Recognition of discharges Electra (CIGRE) No 11, pp 61-98

[11] Nattrass D A 1988 Partial discharge measurement and interpretation IEEE Electric. Insul. Magazine 4 10-23

[12] Laurent C and Mayoux C 1992 Partial discharge: XI. Limitations to PD as a diagnostic for deterioration and remaining life IEEE Electric. Insul. Magazine 8 14-17

[13] Satish L and Gururaj B I 1993 Use of hidden Markov models for partial discharge pattern classification IEEE Trans. Electric Insul. 28 172-82

[14] Hikita M, Kato T and Okubo H 1994 Partial discharge measurements in $\mathrm{SF}_{6}$ and air using phase-resolved pulse-height analysis IEEE Trans. Diel. Electric. Insul. 1 267-83

[15] Gulski E 1995 Digital analysis of partial discharges IEEE Trans. Diel. Electric. Insul. 2 822-37

[16] Cachin C and Wiesmann H J 1995 PD recognition with knowledge-based preprocessing and neural networks IEEE Trans. Diel. Electric. Insul. 2 578-89

[17] Ziomek W, Schlemper H-D and Feser K 1996 Computer aided recognition of defects in GIS Proc. IEEE Int. Symp. on Electric. Insul. (Montreal) pp 91-4

[18] Huecker T 1997 UHF partial discharge expert system diagnosis Proc. 10th Int. Symp. on High voltage Engineering pp 259-62

[19] Contin A, Montanari G C and Ferraro C 2000 PD source recognition by Weibull processing of pulse height distributions IEEE Trans. Diel. Electric. Insul. 7 48-58

[20] Candela R, Mirel G and Schifani R 2000 PD recognition by means of statistical and fractal parameters and a neural network IEEE Trans. Diel. Electric. Insul. 7 87-94

[21] Salama M M A and Bartniaks R 2000 Fuzzy logic Applied to PD pattern classification IEEE Trans. Diel. Electric. Insul. 7 118-23

[22] Hikita M, Yamada K, Nakamura A, Mizutani T, Oohasi A and Ieda M 1990 Measurements of partial discharges by computer and analysis of partial discharge distribution by the Monte Carlo method IEEE Trans. Electric. Insul. 25 453-68

[23] Niemeyer L, Fruth B and Kugel H 1991 Phase resolved partial discharge measurements in particle contaminated $\mathrm{SF}_{6}$ insulation Geasous Dielectrics vol VI, ed L G Christophorou and I Sauers (New York: Plenum) 
[24] Niemeyer L, Fruth B and Gutfleisch F 1991 Simulation of partial discharges in insulation systems 7 th ISH paper 71.05

[25] Niemeyer L 1993 Interpretation of PD measurements in GIS Proc. 8th Int. Symp. on High Voltage Engineering paper 68.04

[26] Gutfleisch F and Niemeyer L 1995 Measurement and simulation of PD in epoxy voids IEEE Trans. Diel Electric. Insul. 2 729-43

[27] Suzuoki S Y, Komori F and Mizutani T 1996 Partial discharges due to electrical treeing in polymers: phase-resolved and time-sequence observation and analysis J. Phys. D: Appl. Phys. 29 2922-31

[28] Morshuis P and Niemeyer L 1996 Measurement and simulation of discharge induced ageing processes in voids Proc. CEIPD (San Francisco)

[29] Schifani R, Romano P and Candela R 2001 On PD mechanisms at high temperature in voids included in an epoxy resin IEEE Trans. Diel Electric. Insul. 8 589-97

[30] Gardiner C W 1985 Handbook of Stochastic Methods (Springer Series in Syergetics vol 13) (Berlin: Springer)
[31] van Kampen N G 1992 Stochastic Processes in Physics and Chemistry

[32] Silverman B W 1986 Density Estimation for Statistics and Data Analysis (Chapman and Hall: London)

[33] Press W H, Flannery B P, Saul S A and Vetterling W T 1992 Numerical Recipes (London: Cambridge University Press)

[34] Efron B and Tibshirani R J 1998 An Introduction to the Bootstrap (New York: Chapman and Hall)

[35] http://www.pd-systems.com/

[36] Gross D W and Fruth B A 1998 Characteristics of phase resolved partial discharge patterns in spherical voids CEIDP98

[37] Pedersen A, Crichton G C and McAllister I W 1995 The functional relations between partial discharges and induced charges IEEE Trans. Diel. Electric. Insul. 2 535-43

[38] Crichton G C, Karlsson P W and Pedersen A 1989 Partial discharges in ellipsoid and spherical voids IEEE Trans. Electric. Insul. 24 335-42 